\title{
The Effect of Layer-by-Layer Building in Selective Laser Sintering on the Performance of Polymer- Based THz Optical Systems
}

\section{Gabriel Moagar-Poladian ( $\boldsymbol{\sim}$ gabriel.moagar@imt.ro)}

National Institute for research and Development in Microtechnology - IMT Bucharest

Catalin Tibeica

National Institute for research and Development in Microtechnology - IMT Bucharest

\section{Research Article}

Keywords: THz lens, additive manufacturing, electromagnetic simulations, step-like structures in additive manufacturing, self-aligned THz optical system

Posted Date: October 20th, 2021

DOl: https://doi.org/10.21203/rs.3.rs-959517/v1

License: (c) (i) This work is licensed under a Creative Commons Attribution 4.0 International License.

Read Full License 


\title{
THE EFFECT OF LAYER-BY-LAYER BUILDING IN SELECTIVE LASER SINTERING ON THE PERFORMANCE OF POLYMER-BASED THz OPTICAL SYSTEMS
}

\author{
Gabriel MOAGĂR-POLADIAN ${ }^{(*)(1)}$, Cătălin TIBEICĂ $\breve{(1)}^{(1)}$ \\ ${ }^{(1)}$ National Institute for Research and Development in Microtechnology - IMT Bucharest \\ Str. Erou Iancu Nicolae 126A, 077190, București-Voluntari, ROMANIA \\ (*) E-mail: gabriel.moagar@imt.ro
}

\begin{abstract}
Additive manufacturing technologies have reached a point where ready-to-use items are directly produced from a PC-stored data file. Among these technologies, selective laser sintering has become a mature technology able to fabricate complex geometric structures using a variety of materials. Despite the versatility of this technology, it also has some drawbacks. One of those limitations, of major concern for building optical elements, is the step-like structure of the surface specific to the layer-by-layer building. In our paper, we present extensive full-wave electromagnetic calculations that consider the effect of those steps on the optical behaviour of refractive lenses made for the $\mathrm{THz}$ spectral domain. Our results show that at least up to $1.5 \mathrm{THz}$, the additivelly manufactured, stepwise lens behaves very close to its ideally smooth surface counterpart.
\end{abstract}

\section{Keywords}

THz lens, additive manufacturing, electromagnetic simulations, step-like structures in additive manufacturing, self-aligned THz optical system

\section{Introduction}

With the advent of more powerful THz radiation sources, such as those based on fs-laser, THz quantum cascade lasers, $\mathrm{THz}$ parametric oscillators, free electron lasers in addition to more performant detectors, the $\mathrm{THz}$ spectral domain has become a field of maximum interest for many imaging applications such as medicine [1-5], atom probe tomography [6], spectroscopy [7-9], astronomy [10-12], defense and security [13-15], quality control [16-18] to name only a few.

In all these fields, imaging of $\mathrm{THz}$ radiation is a key component and therefore $\mathrm{THz}$ optics plays a central role [19-23]. Developing materials that are transparent in this window of the electromagnetic spectrum and are easily machined to high performance geometries is of huge importance. In the last decade, several polymeric materials have been demonstrated to be used for various types of optical elements [24-31].

Because of so many applications, finding a way to rapidly and cost effectively produce optical elements for the $\mathrm{THz}$ imaging systems plays a key role. For that to happen, a successful technology should allow processing in a low-cost manner and ensure achieving of complex geometrical shapes for increased part functionality. Such a technology is selective laser sintering (SLS) of polymers. SLS fabrication is a layer-by-layer process giving rise to a stair-like (stepwise) structure of the object surface. The effect of "stairs" resulting from this process on the post-lens optical field distribution can hamper the appropriate optical behaviour of $\mathrm{THz}$ lenses made this way.

It is the aim of this paper to show, by extensive electromagnetic simulations, that the stepwise structure of the surface has a reduced effect especially for the lower frequencies part of the $\mathrm{THz}$ domain, that is for frequencies up to 1.5 THz.

The paper is structured as follows. In section II we present preliminary considerations regarding the PA2200 material, which is a material largely used in polymer SLS systems. In section III we present extensive electromagnetic simulation data of the optical behaviour of $\mathrm{THz}$ lenses made of this material. The paper ends with the Discussions and Conclusion sections, respectively. 
Throughout this paper, by optical / optics we will refer solely to the THz range with no connection to the visible spectrum.

\section{Material properties and preliminary considerations}

The PA12 (PA2200) material is known [32] to have an absorption coefficient of about $1 \mathrm{~cm}^{-1}$ at $300 \mathrm{GHz}$, making it an interesting choice when considering additive fabrication of refractive optical elements for the respective spectral range. In the following we will consider this material as regards the simulation of the refractive optical element behaviour, the optical element (specifically spherical lens) being made by SLS. The main problem that arises here is the stair-like structure of the lens curved surface. We will compare the behaviour of the stair-like lens with that of a similar lens that has a smooth surface. The simulations are made by full-wave electromagnetic finite element analysis. We consider two extreme frequencies: $100 \mathrm{GHz}$ (where the material has low absorption) and, at the higher part, $1 \mathrm{THz}$. While PA12 is not transparent at $1 \mathrm{THz}$, we have chosen this frequency just to prove that even if the stair-like surface might create difficulties in focusing on EM radiation, the additively manufactured lens still behaves appropriately. To make simulations at $1 \mathrm{THz}$, we considered the material as being transparent and studied the way the stairs and the edges on the lens' curved surface influence the focusing properties.

The literature has many references indicating various values for absorption coefficient of the PA12 material in the $\mathrm{THz}$ range. Such a dispersion of the values is normal since there are many blends of nylon 12 used for applications. Each of these compositions has a certain absorption spectrum and a specific refractive index according to its ingredients. As it is known, absorption in the $\mathrm{THz}$ spectrum strongly depends on the vibrations of the molecular chains and of the individual molecular (functional) groups. Changing this environment causes change of the $\mathrm{THz}$ absorption spectrum. Moreover, the thermal history to which the material is subjected is also an important factor that contributes to the variations in the THz refractive index and absorption spectrum. The percentage of the crystalline/semi-crystalline component of nylon, the change of length of the molecular chains during the specific thermal process contribute all to the change of the molecule vibrational spectrum and hence of its $\mathrm{THz}$ absorption spectrum. This could be the reason for differences in absorption coefficient between [32] and [33], for example.

Moreover, there are several thermoplastic polymers transparent in the $\mathrm{THz}$ domain having refractive index values ranging from 1.46 (the TPX material) to more than 1.7. Thus, for simulations we consider a value $\mathrm{n}_{\text {Lens }}=1.516$ for the refractive index. We have chosen an intermediate value situated between the low value specific to the TPX (1.46) polymer and the high value specific to the PA2200 (1.64) one [32].

\section{Simulation of SLS-made THz lenses}

III.1 Staircase-like lens (stepwise lens)

We consider a plano-convex spherical lens with the following dimensions: Lens diameter, $\mathrm{D}_{\text {Lens }}=40 \mathrm{~mm}$; Lens sagitta, $\mathrm{s}_{\text {Lens }}=5 \mathrm{~mm}$; Lens thickness, $\mathrm{t}_{\text {Lens }}=10 \mathrm{~mm}$. Figure 1 depicts the elements of the lens.

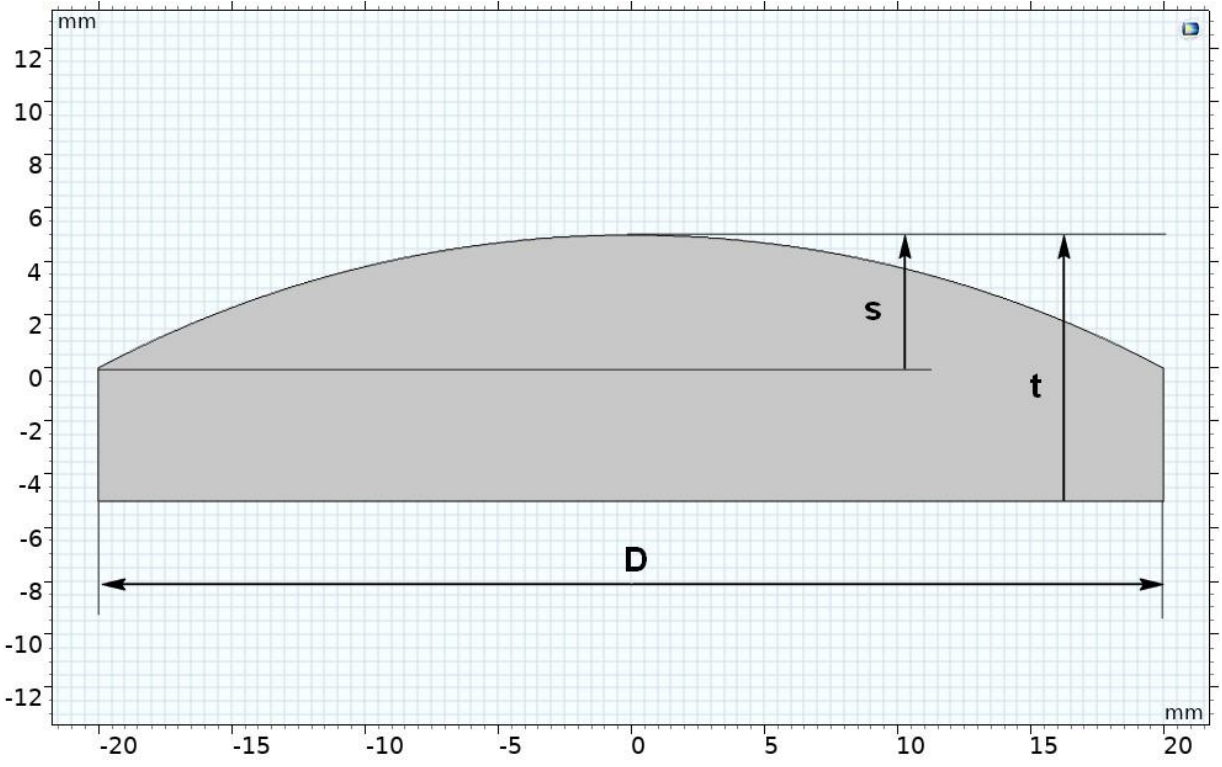

Figure 1 - The geometrical elements of the lens. 
For the printed layer thickness, we consider the value $\Delta \mathrm{z}=0.1 \mathrm{~mm}$, with the apex of the lens being oriented upward. In other words, the lens is built starting with the planar base, this planar base being normal to the SLS building direction and normal to the optical axis of the lens.

The finite element simulations are made by using the COMSOL Multiphysics software for solving the Maxwell equations. The discretization step for the mesh is $\mathrm{N}_{\text {Mesh }}=\lambda / 8$, where $\lambda$ is the radiation wavelength.

The amplitude of the incident electric field was taken equal to $1 \mathrm{~V} / \mathrm{m}$. The simulations are performed in the linear regime, i.e., the electromagnetic properties of the lens material do not depend on the incident radiation intensity.

The axial section of the stepwise lens is presented in figure 2 together with the method of stepwise lens model construction. We considered the ideal lens and sectioned it with parallel planes normal to the optical axis and distanced 100 microns apart, this distance representing the thickness of the building layer of the machine. Then, after this sectioning, we built the steps in the model at the intersection between the cutting planes and the ideal contour of the lens.

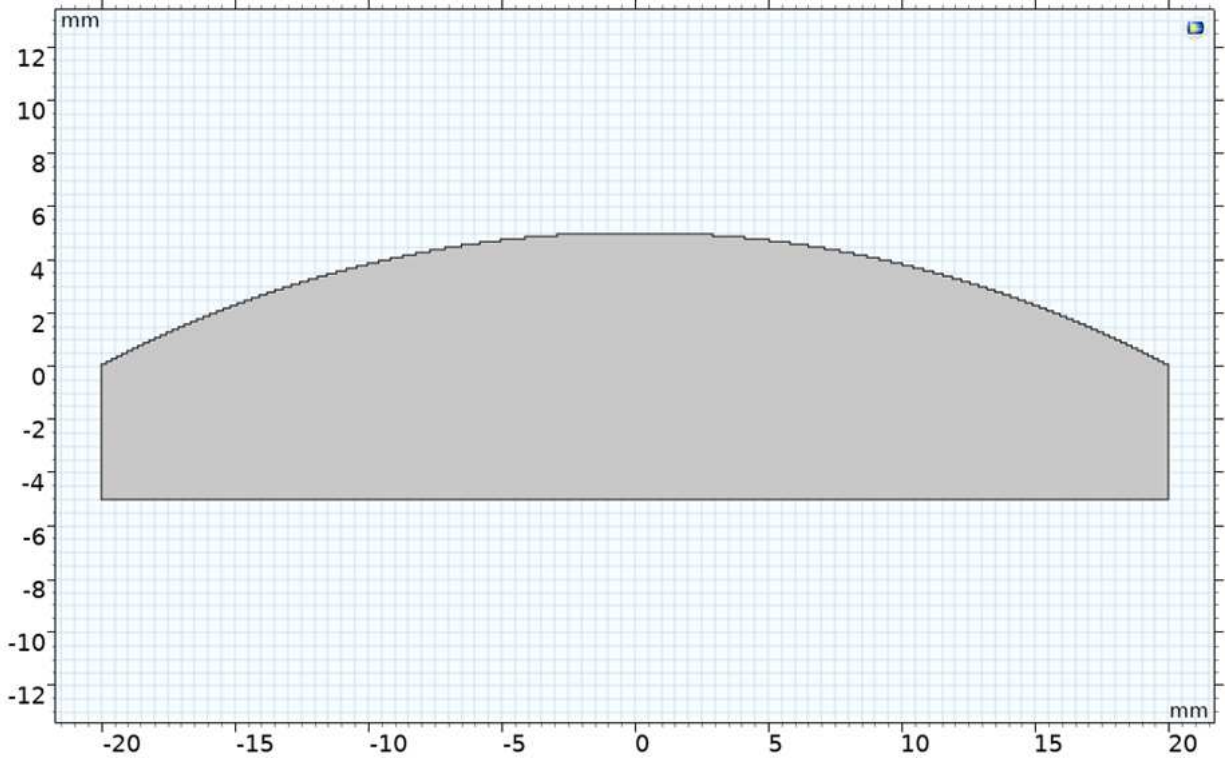

a)

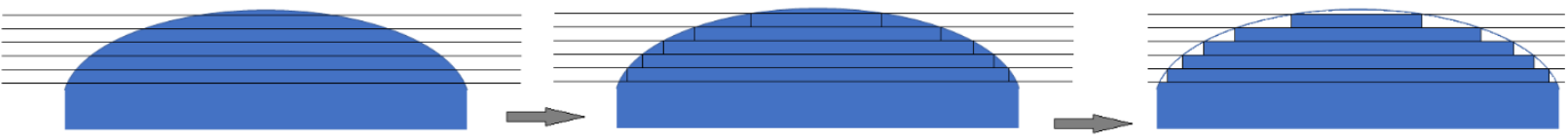

b)

Figure 2 -The structure of the stepwise lens. a) side profile of the stepwise lens modelled for FEM simulation; $b$ ) the way by which the stepwise structure of the lens was obtained.

Simulations were performed considering an incoming plane wave at different incident angles, in angular steps of $0.5^{\circ}$, which is very close to the Rayleigh separation criterion $\theta_{\mathrm{R}}$ for the given lens parameters $(\mathrm{D}-$ diameter, $\lambda-$ wavelength):

$$
\theta_{\mathrm{R}}[\mathrm{rad}] \approx \sin \left(\theta_{\mathrm{R}}\right)=1.22 \lambda / \mathrm{D}, \quad \theta_{\mathrm{R}}=0.52^{\circ}
$$

The wavelength considered is 300 microns, corresponding to a frequency of $1 \mathrm{THz}$. We have chosen this value because we expect that at shorter wavelengths the differences between stair-cased and ideally smooth lenses respectively to be more easily visible.

A comparison between the stepwise and smooth lenses, respectively, is presented in figure 3, where the focal point coordinate is described as a function of the incidence angle for these two types of lenses.

As a double-check, we have also performed the analytical computation of the focal length for the smooth lens using the thick lens formula from geometrical optics. The analytical calculation and the value obtained from simulations are practically identical. The focal plane, considered as the distance where the electric field reaches its maximum for a normal incident wavefront $\left(\theta=0^{\circ}\right)$, has, for both lenses (smooth and stepwise), a value of $153 \mathrm{~mm}$. More detailed simulation results for this case can be found in figures S1 - S4 in the Annex. 


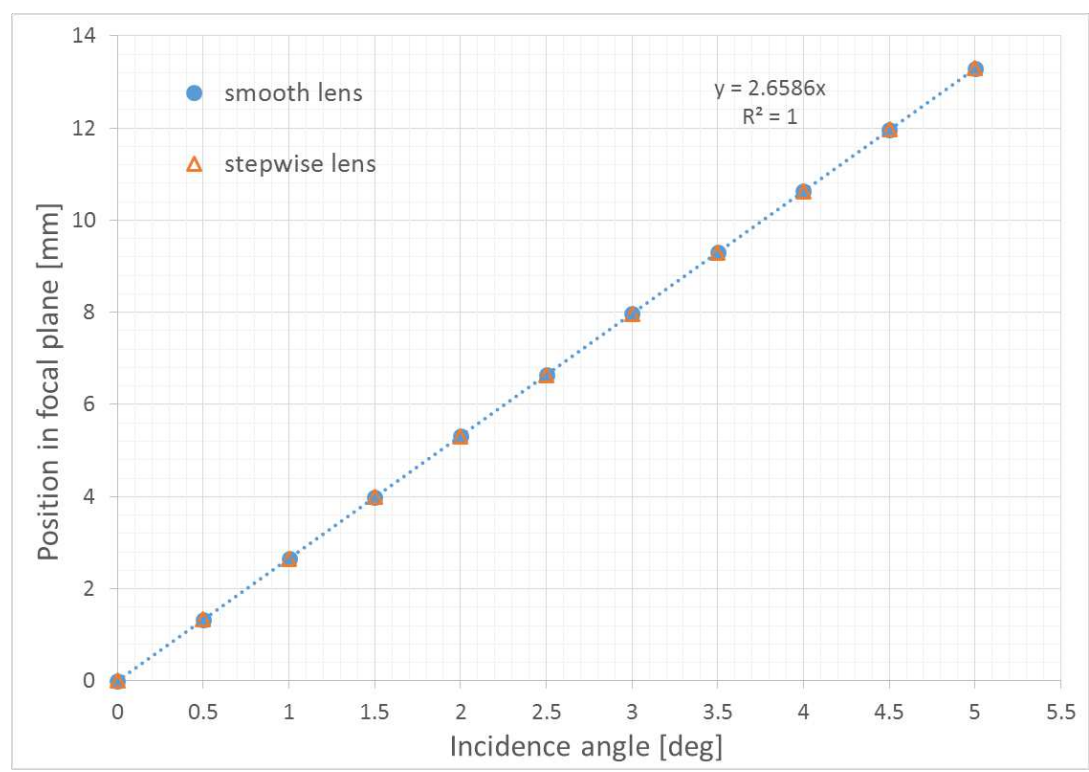

Figure 3 - Focus point coordinate in focal plane as function of incidence angle (smooth vs. stepwise lens). The equation in the figure represents the fitting of the simulation points, while $R$ is the linear regression coefficient. Frequency of the radiation is equal to $1 \mathrm{THz}$.

The results show that even at $1 \mathrm{THz}$ the focal length of the two lenses (stepwise and ideal) are equal for all incidence angles.

Results from figures 3 and S1-S4 demonstrate that the stepwise lens behaves almost like the ideal one at $1 \mathrm{THz}$. This similarity is even better at longer wavelengths (lower frequencies). As frequency goes higher, the graph oscillations around the maximum in figures S2 and S6 go higher for the stepwise lens. This is expected since at shorter wavelengths the diffraction cannot smoothen the radiation intensity distribution as easily as in the case of longer wavelengths and the effect of the step-like structure of the lens becomes more pronounced.

Figure 4 shows the value of the electrical field maximum in the focal point as a function of the incidence angle.

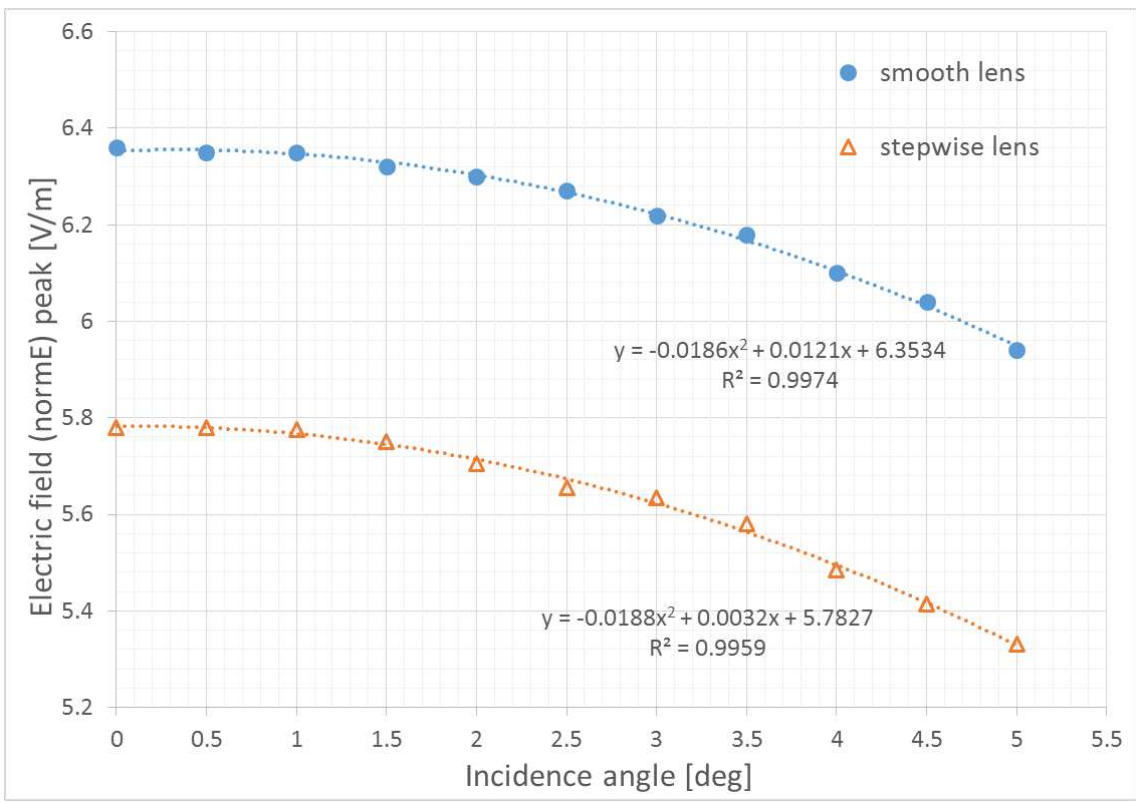

Figure 4 - Electric field maximum in focal plane as function of incidence angle (smooth vs. stepwise lens). The equation in the figure represents the fitting of the simulation points, while $R$ is the linear regression coefficient. 
We notice from figure 4 that the two lenses behave almost identically, within a $1 \%$ margin, as regards the dependence of the electric field maximum on the incidence angle; the only difference lies in the magnitude of the maximum electric field, which is around $10 \%$ lower for the stepwise lens.

More information about the behaviour of the stepwise lens at three different wavelengths at normal incidence is presented in section A.2 of the Annex.

An important comparison between the two types of lenses is about the distribution of the electric field of the focused electromagnetic wave in the focal plane. Figure 5 represents the respective comparison at $100 \mathrm{GHz}$ while figure 6 shows the same comparison at $1 \mathrm{THz}$.
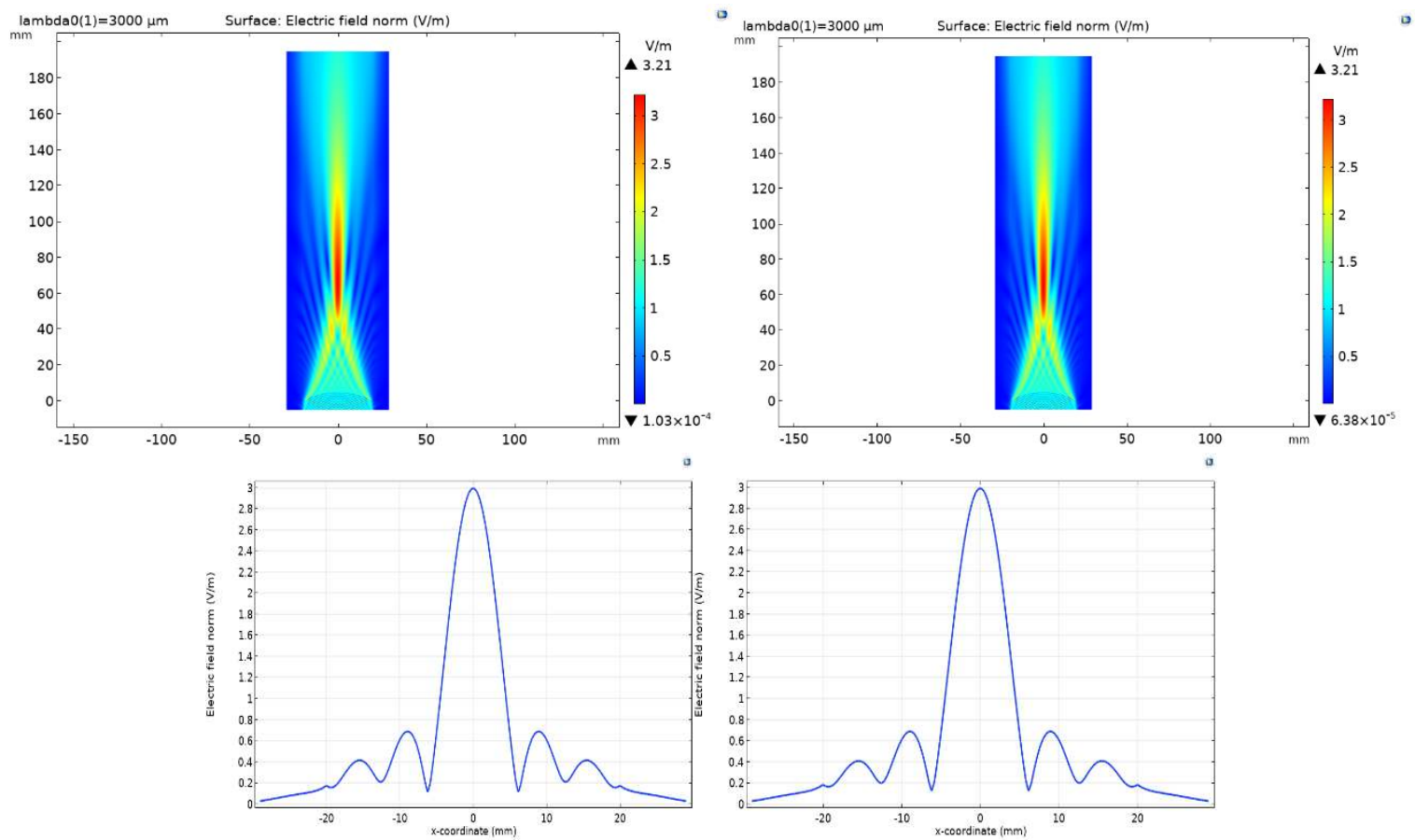

Figure 5 - Comparison between stepwise and ideal lens at $100 \mathrm{GHz}$. Top left: electric field distribution for the stepwise lens; Top right: electric field distribution for the ideal lens; Bottom left: distribution of electric field in the focal plane;

Bottom right: distribution of electric field in the focal plane.

As it can be noticed from figure 5, there is no difference between the stepwise and ideal lens as regards the electric field distribution at $100 \mathrm{GHz}$.

We must underline here that it could be debatable whether we consider acceptable a $\lambda / 8$ discretization when the $\mathrm{THz}$ radiation wavelength at $100 \mathrm{GHz}$ is $3 \mathrm{~mm}$ and the build step of the lens is $0.1 \mathrm{~mm}$ or not. In this discretization, we have 5 nodes per step height. In order to check if there is any significant error, we repeated the simulation with 10 times denser discretization, with an element size equal to $\lambda / 8$. The comparative results are shown in table 1 . The element size was that used for the simulation in the case of the $1 \mathrm{THz}$ radiation. 
Table 1 - Comparison between electric field distribution at $100 \mathrm{GHz}$ for the two discretization cases

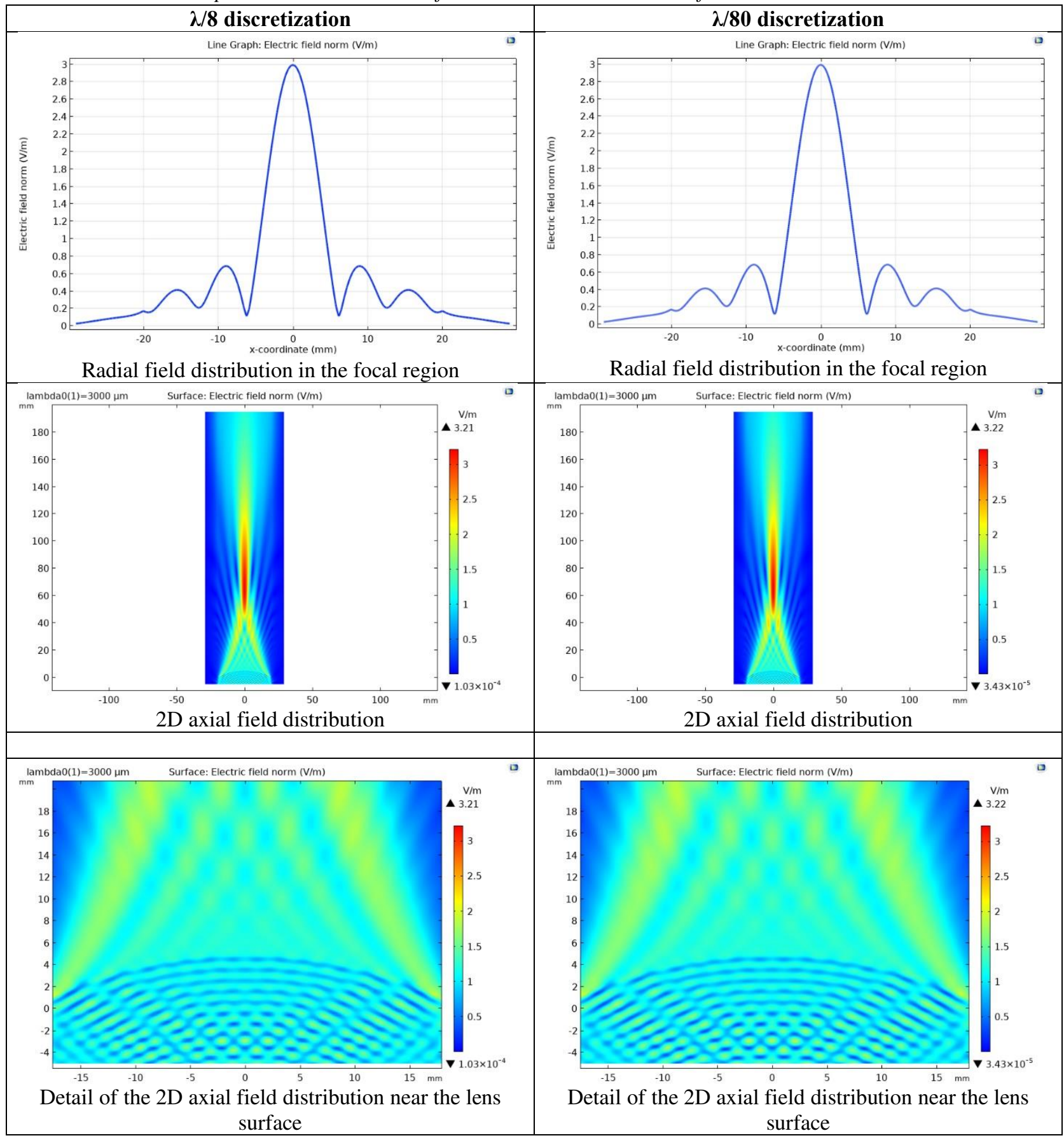

As it can be observed from table 1 , the difference between the two field maxima is $0.3 \%$. This is a very small difference and we may consider that the $\lambda / 8$ discretization gives correct results. 

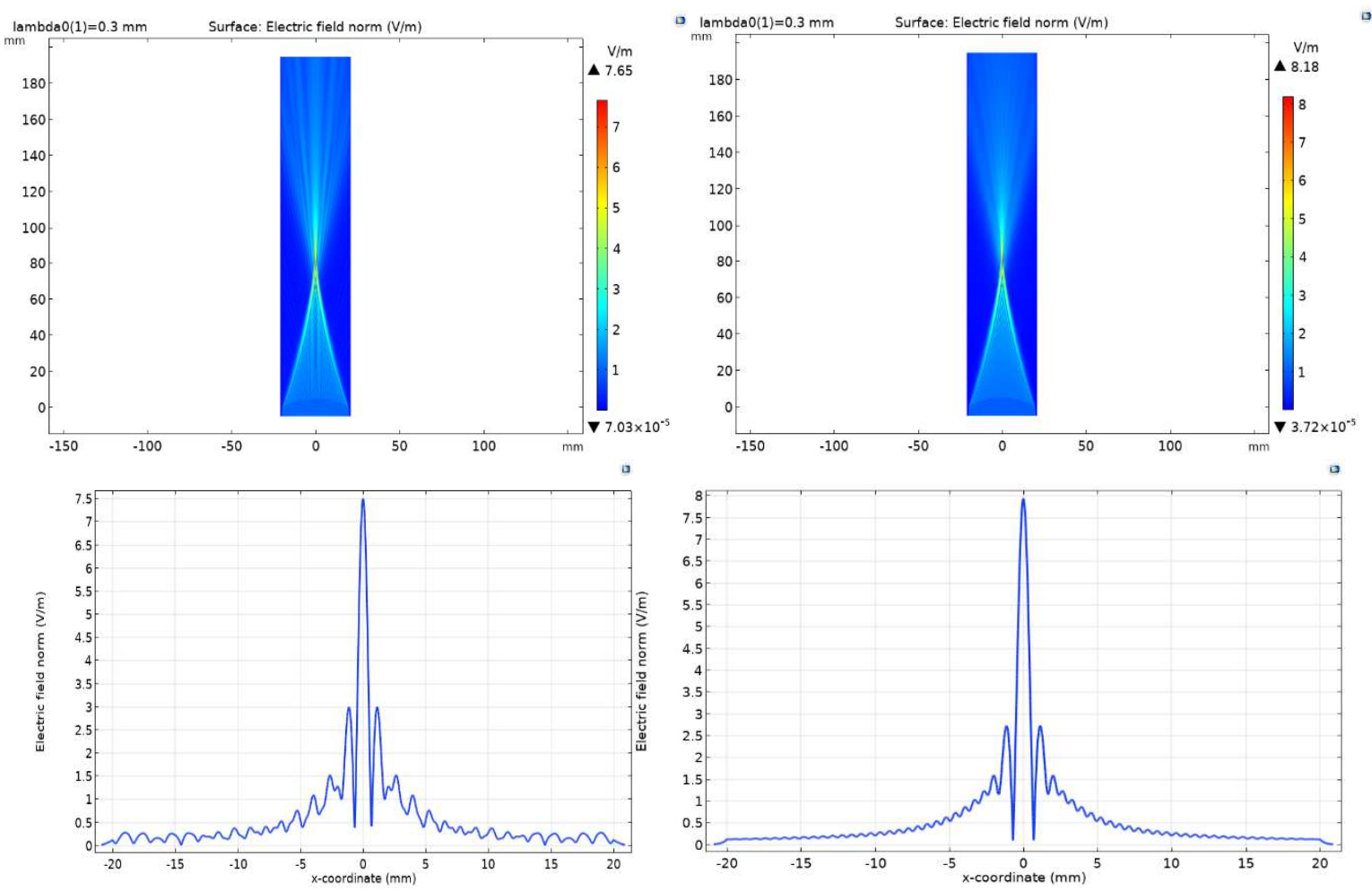

Figure 6 - Comparison between stepwise and ideal lens at $1 \mathrm{THz}$. Top left: electric field distribution for the stepwise lens; Top right: electric field distribution for the ideal lens; Bottom left: distribution of electric field in the focal plane; Bottom right: distribution of electric field in the focal plane.

Three observations must be made in the case of higher frequencies (e.g., $1 \mathrm{THz}$ ). First, the intensity of the maximum electric field is higher as compared to the $100 \mathrm{GHz}$ case. Secondly, the stepwise lens has a noisier field distribution around the maximum than the ideal lens. This is because diffraction cannot smoothen as effectively as for lower frequencies the uneven distribution due to the stepwise structure. Thirdly, the maximum value of the electric field for the stepwise lens is slightly lower than for the ideal lens, namely $7.5 \mathrm{~V} / \mathrm{m}$ compared to $8 \mathrm{~V} / \mathrm{m}$. However, even at 1 $\mathrm{THz}$, the stepwise lens behaves well and relatively close to the ideal one. It is seen from figure 6 that the half-width of the simulated focal spot is around 700 microns $(\approx 2.3 \lambda)$ which compares quite well to the ideal diffraction limited spot of $1.22^{*} \lambda^{*}(\mathrm{f} / \mathrm{D})$, with ' $\mathrm{f}$ ' standing for the focal length while is ' $\mathrm{D}$ ' standing for the lens diameter.

We may thus affirm that the SLS technique can fabricate THz lenses with good performances up to $1 \mathrm{THz}$. On the other hand, if only focusing is needed instead of image forming, then the frequency range can be extended up to 3 THz. Moreover, using thinner building layer (e.g., 60 microns) and newer systems, like the EOS GmbH SLS systems that can achieve features of 200 microns compared to 400 microns features obtained with our polymer SLS machine Formiga P100 (here we speak about lateral resolution), optical elements for imaging can be obtained for frequencies up to $3 \mathrm{THz}$, provided that the polymer is transparent in this spectral range.

\section{2 Lens doublet}

Next, we consider two identical plano-convex spherical lenses that form a simple THz optical system. A system of two lenses is the simplest testbed to determine how stepwise lenses affect an imaging system. The following configurations were simulated, as depicted in figure 7 :
A. Lenses only
B. Lenses with a support barrel
C. Lenses with barrel and a front aperture

The analysis (comparison) is performed for an as printed optical system; therefore, the lenses are all of stepwise type. The model consists of a lens doublet together with its barrel housing, made in one piece from the same material. The 
barrel serves as a frame for the self-alignment of lenses. In other words, the set of lenses and their mechanical support are all additively manufactured. Such a fabrication approach is useful when someone needs to align several optical components (lenses) for making an optical system. This eliminates the need for cumbersome and time-consuming alignment procedures and represents one of the advantages that SLS brings to the THz optics industry. We additionally suppose that the powder embedded between the two lenses during the building process is removed from there so that there is only air between the lenses.

The construction parameters are:

$$
\begin{aligned}
& D_{\text {Lens } 1}=D_{\text {Lens } 2}=50 \mathrm{~mm}, \\
& \mathrm{~s}_{\text {Lens } 1}=\mathrm{s}_{\text {Lens } 2}=3.5 \mathrm{~mm}, \\
& \mathrm{t}_{\text {Lens } 1}=\mathrm{t}_{\text {Lens } 2}=7 \mathrm{~mm},
\end{aligned}
$$

Distance between lenses, $\mathrm{d}=22 \mathrm{~mm}$,

Barrel thickness $=6 \mathrm{~mm}$,

Front aperture diameter $=48 \mathrm{~mm}$,

Refractive index, $\mathrm{n}=1.516$

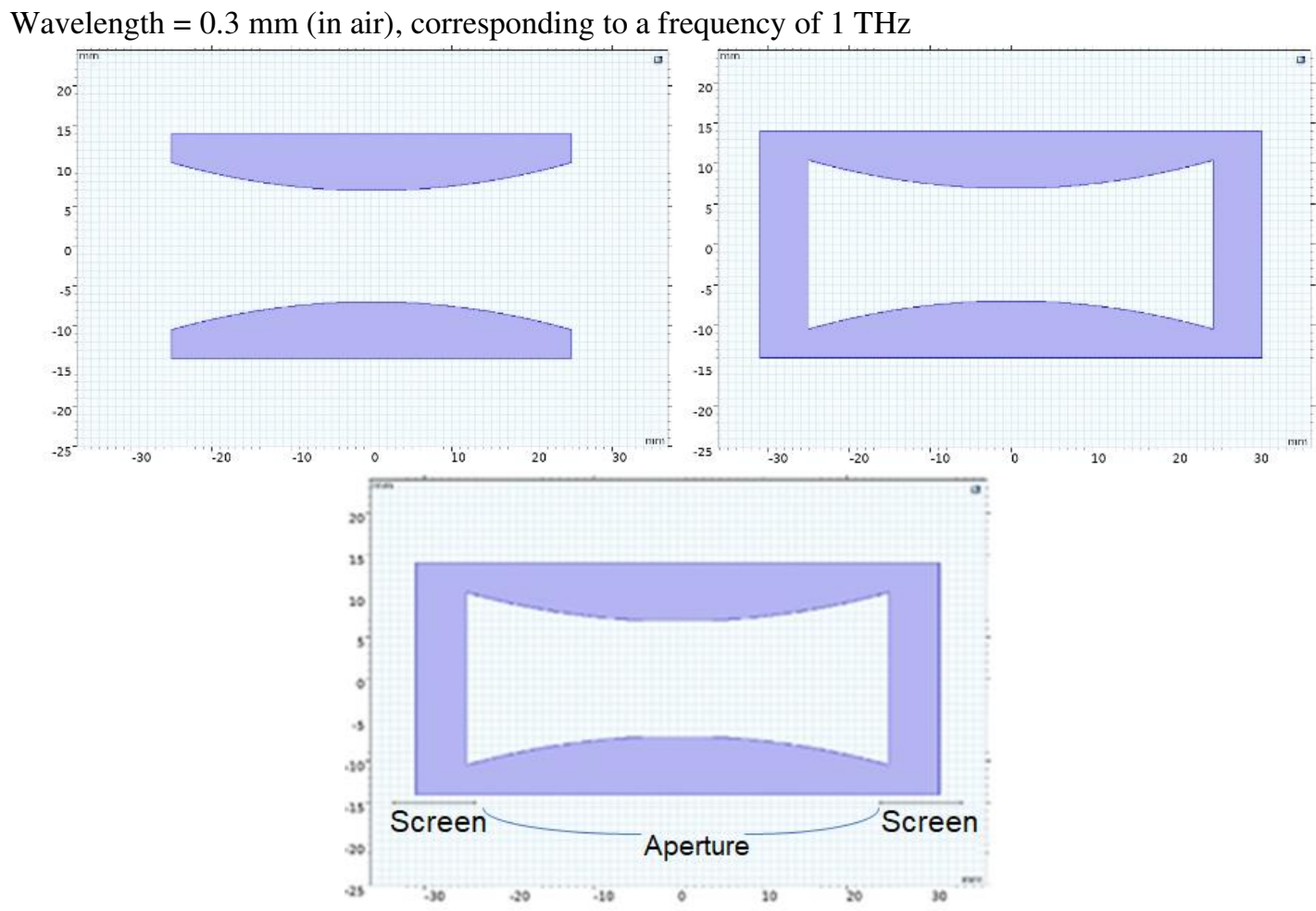

Figure 7 - Models considered for simulations. Top left (model A) - lenses only; Top right (model B) - lenses with a barrel; Bottom (model C) - lenses with a barrel and front aperture.

The simulation results showing the electric field distribution in the focal plane, for the three models (A, B and C), are shown in figure 8 . 

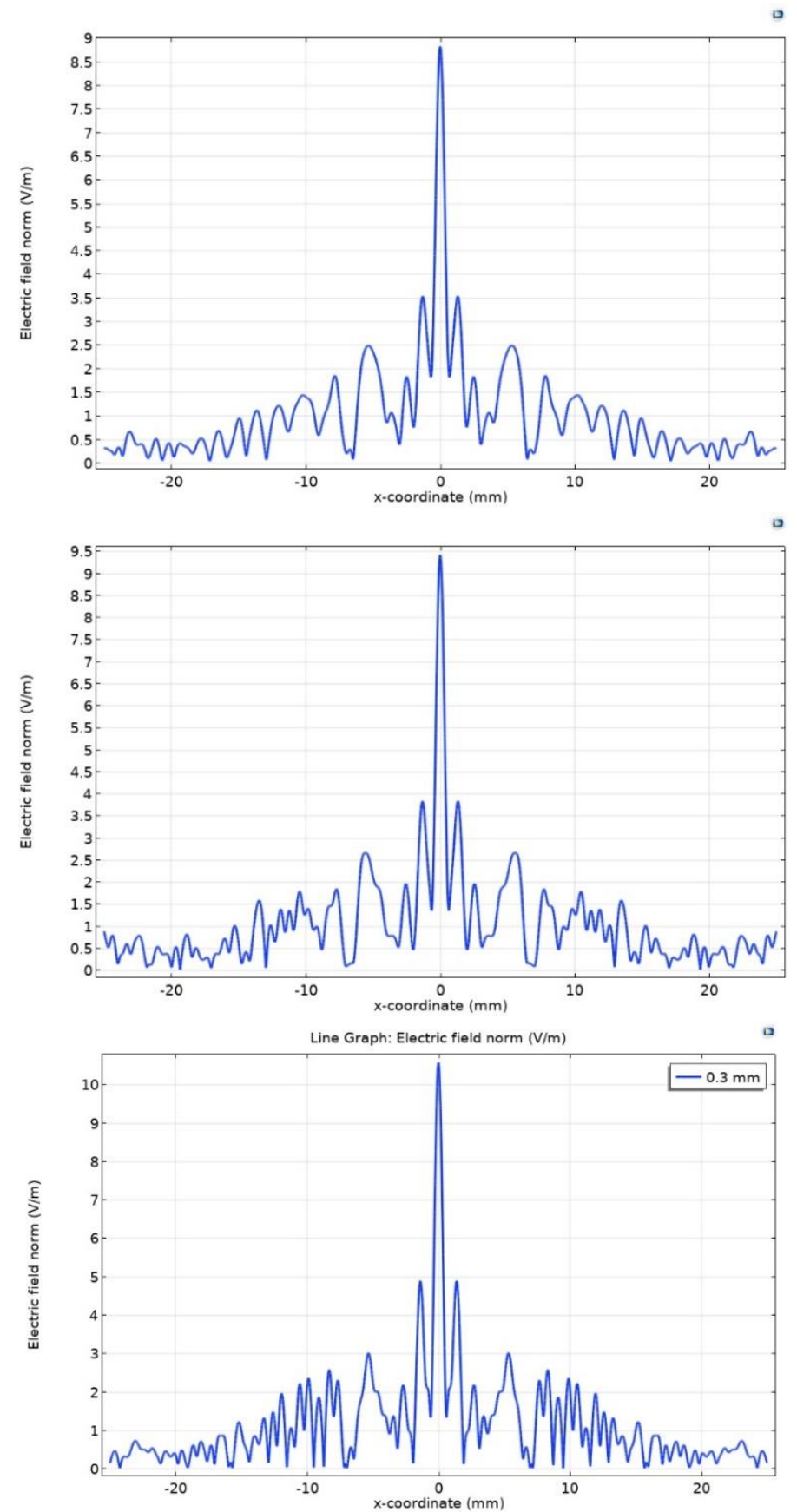

Figure 8 -Electric field distribution of the wave in the focal plane. Top - lenses only; Centre - lenses with a barrel; Bottom - lenses with a barrel and front aperture.

From figure 8 we notice that the barrel's wall introduces noise in the electric field distribution, which is due to reflections and refractions. Part of the radiation coming from the barrel's wall interferes with the radiation coming directly from lenses and gives a noisier distribution in the lateral wings of the focal plane as compared to the doublet without barrel. This phenomenon is amplified by the presence of the front aperture, which gives rise to additional diffraction on the aperture's margins. On the other hand, the value of the central maximum increases for the case of the barrel and barrel and aperture, respectively.

Overall, the doublet focuses the radiation on a good manner, proving the feasibility of fabricating the entire, selfaligned optical system including its mechanical frame by 3D Printing. At lower frequencies the behaviour is even better, the longer wavelength smoothening the noisier wings. However, at lower frequencies, the aperture of the barrel will increase diffraction at its margins but moving the barrel wall further with respect to the optical axis may reduce the 
diffraction influence. It is important to notice that if the barrel does not have a continuous lateral surface of the cylinder and, instead, it is formed of only 3-4 rods, then the effect of the barrel is significantly diminished.

\section{III.3 Analysis of image formation}

III.3.1 Single point image formation

In the following we analyse the way the stepwise lens forms an image. We start with the imaging of a single point. This point is situated first on the optical axis of the lens, then it lays in the same plane but radially displaced up to $10 \mathrm{~mm}$ in steps of $1 \mathrm{~mm}$. The point source is a pinhole that has a diameter of $0.5 \mathrm{~mm}$ (almost $1.5 * \lambda$, where $\lambda$ is the radiation wavelength). We perform the simulations for two lenses of similar geometries, but one of them has a smooth surface and the other one is a stepwise lens. Figure 9 shows the set-up. The simulation parameters are mentioned in the Annex at section A.3 . An electromagnetic THz plane wave is incident on the pinhole, coming from the left.

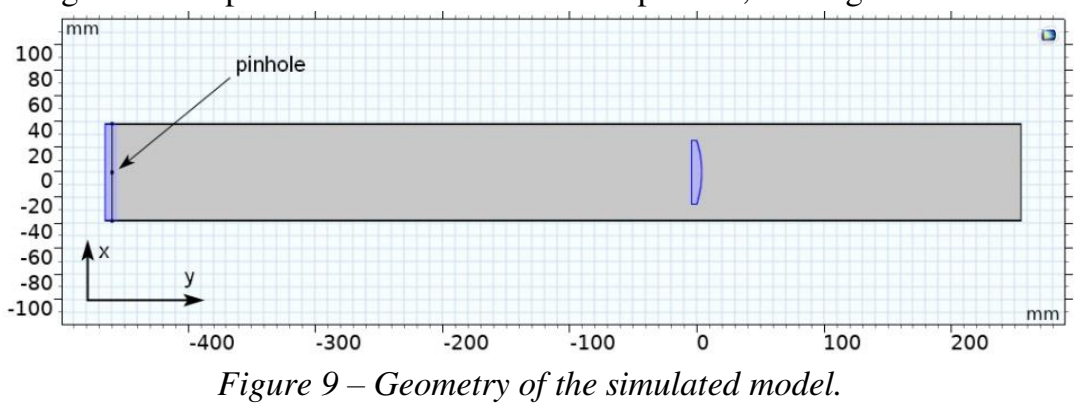

The field distribution immediately after the pinhole is shown in the Annex in figure S.7, while figure S.8 depicts the point image formation for the stepwise lens for the two positions (optical axis and, respectively, radially displaced at $10 \mathrm{~mm}$ ). We notice in figure S.8 that the stepwise lens focuses the point object to a point image for each of the pinhole positions.

To compare the quality of images, a transversal section through the image plane shows the field amplitude distribution in the image plane, as depicted in figure 10 for the stepwise and smooth lens, respectively.

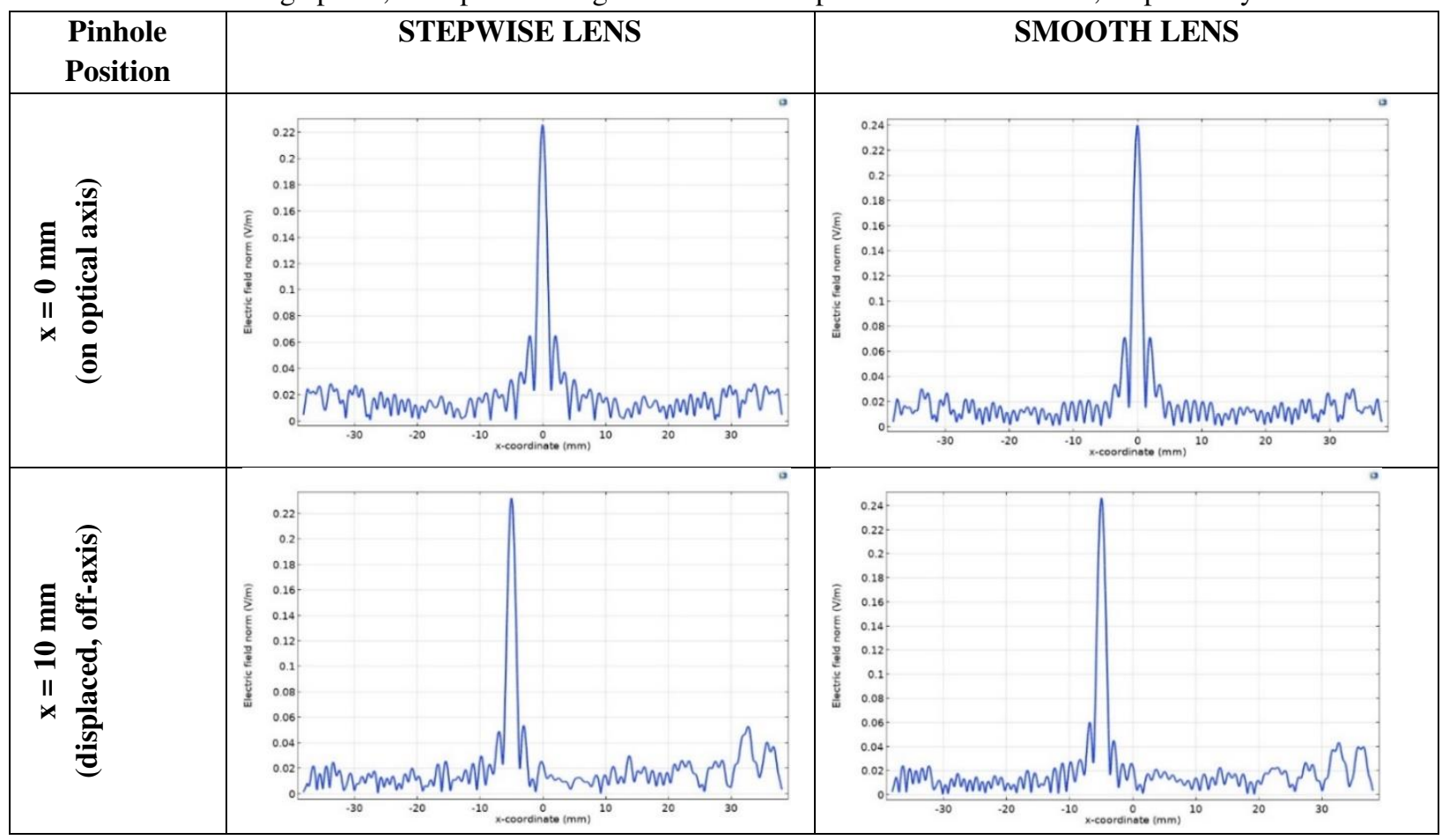

Figure 10 - Electric field distribution in the image plane. Top left: image object at $x=0 \mathrm{~mm}$ for stepwise lens; Top right: image object at $x=0 \mathrm{~mm}$ for smooth lens; Bottom left: image object at $x=10 \mathrm{~mm}$ for a stepwise lens; Bottom right: image object at $x=10 \mathrm{~mm}$ for smooth lens. 
It can be noticed that for each point object position its image is a point-like one and it is situated at the same position for both lenses, the only differences between the electric field distributions of the two lenses being the values of the central maximum (10\% lower for the stepwise lens) and the noisier lateral wings for the stepwise lens. Figure 11 depicts the relation between the position of the object point and that of the image point for the two lenses. As it can be seen, they both have a linear dependence and the difference between their linear regression coefficients is negligible $(0.01$ $\%)$. The difference in slope is less than $1 \%$.
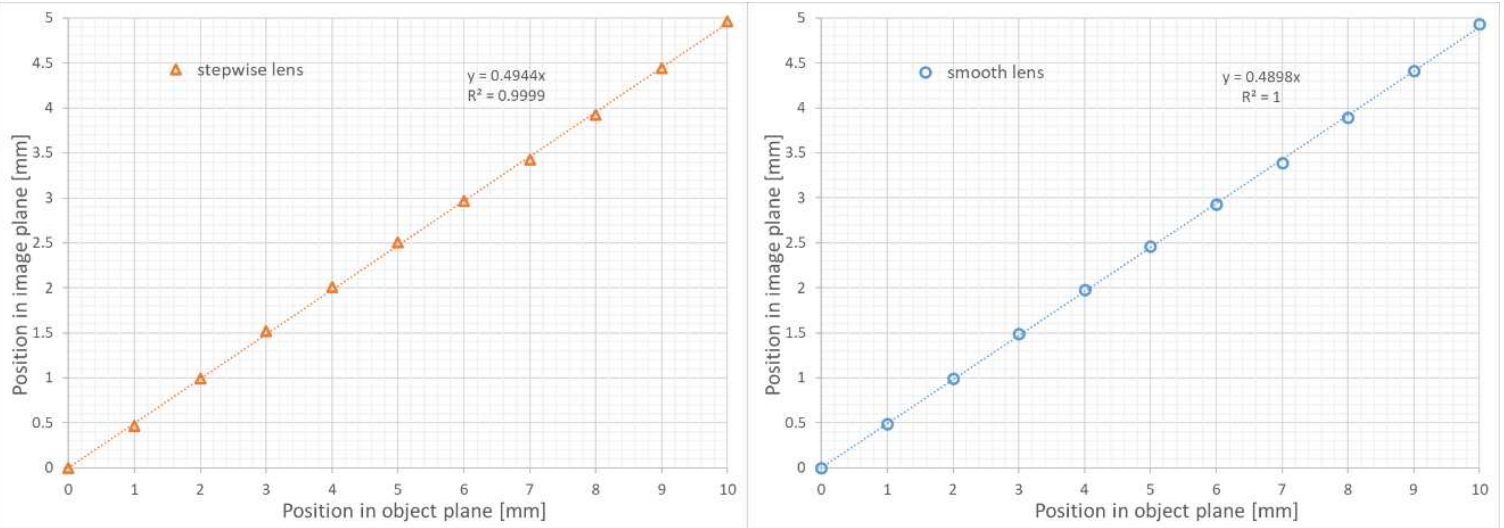

Figure 11 - Relation between point position in object plane and point position in image plane, stepwise lens (left) vs smooth lens (right)

III.3.2 Multipoint image formation

An object has a certain spatial extension; hence it is useful and important to study the image formation for a multipoint source for assessing the quality of the lens. We mention that in our simulation we consider monochromatic, coherent $\mathrm{THz}$ radiation, although interference phenomena are expected when several sources coherently superpose their waves.

We consider a number of eight point-like sources distributed along the radius and spaced at $0.5 \mathrm{~mm}$ from each other. This distance between neighbour points is small enough and is comparable to the radiation wavelength (0.3 mm).

A comparison of the radial distribution of the field for the two lenses is depicted in figure 12, while detailed simulation results are shown in figures S.9 and S.10 of the Annex. As it is expected, each of the lenses performs image inversion (i.e., the image is inverted with respect to the object). The radial distribution shows good similarity between the two lenses as it can be seen from figure 12. It further shows the feasibility of making THz lenses by using SLS on polymer technology.

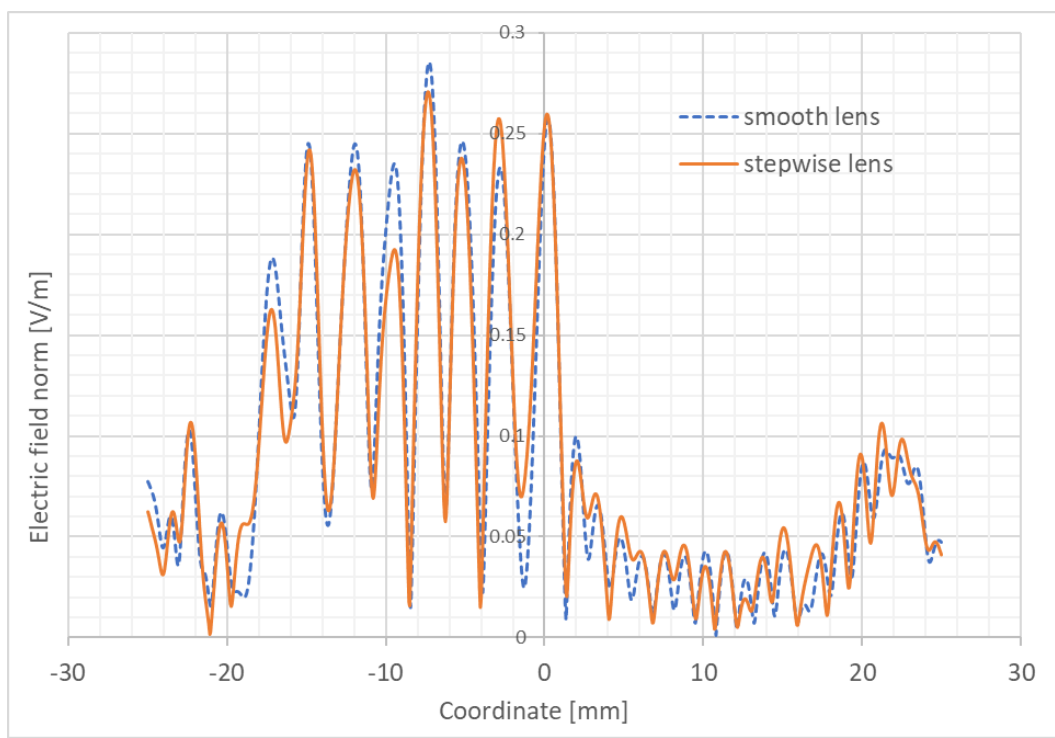

Figure 12 - Comparison of electric field distribution in the image plane for the stepwise lens vs smooth lens. 
As can be seen from figure 12, the two distributions are superposing in a satisfactory manner, thus demonstrating the feasibility of making THz lenses by using SLS on polymer technology that are comparable to their smooth curved surface counterparts.

\section{Discussions}

Except of the stepwise structure specific to additive manufacturing building style that was exhaustively considered above, there also other aspects that have to be taken into account.

First, it is important to underline that the effect of surface steps is reduced if the lens material has a low refractive index in the THz range, which is obvious since the difference in optical paths between two consecutive steps is lower when a lower index material is used.

A special problem that must be taken into consideration is the transparency of the material used for making the THz lenses. At $800 \mathrm{GHz}$, nylon is still transparent [33], but nylon used for SLS is a blend of several substances / additives to make it suitable for the laser sintering process. Those additives change the absorption spectrum, introducing some absorption bands at higher frequencies and thus PA2200 is suitable only for frequencies below $500 \mathrm{GHz}$. In this case, the PA12 additively manufactured lens can be used as a mould for other polymer materials that are more transparent in the $\mathrm{THz}$ range at least for frequencies above $500 \mathrm{GHz}$. The mould is made by a double-inversion technique. In the first step of this technique, micronized gypsum powder paste (water mixed) or other casting material used in additive manufacturing (e.g., Gold Star XL) is used to create a first negative replica of the PA12 lens. The gypsum grain size (average size of 2 microns or less) is good enough to accurately reproduce the 100 microns steps specific to polymer based SLS. Then, in a second step, the dried gypsum part is used as a mould for casting the thermoplastic polymer that has a better transparency in the $\mathrm{THz}$ range. By better transparency we understand not only the small value of the absorption coefficient but also the spectral width of the transparency range. TPX, HDPE (high density polyethylene) or Teflon could be such choices for frequencies from $100 \mathrm{GHz}$ to $1.5 \mathrm{THz}$ [34]. Moreover, the gypsum surface may be smoothened by slightly polishing it with a soft material. In this way, the resulting, stair-less, final optical element will perform even better than its initial PA2200 counterpart. This procedure is harder to be carried out when considering a full, self-aligned optical system. In such a case, this could be achieved only if the entire geometry of the self-aligned system allows mould replication without the destruction of the original, additively manufactured one. It is also important to notice that smoothening slightly changes the geometry of the lens. CAD design must take into account this variation and correct it in order to obtain the final lens product at its desired shape.

A particular aspect of the low loss materials is that diffractive optics (in the THz range) can be made with high efficiency of transmission. In the case of metal-based diffractive optics, a part of the radiation is absorbed by the metal itself, causing signal loss that it is unwanted, especially when trying to image low-power $\mathrm{THz}$ signals. $\mathrm{THz}$ transparent polymers have no such limitation, and thus can be considered as a better candidate for making THz optical elements, either refractive or diffractive. A diffractive Fresnel lens made of PA2200 was already described in [32]. Moreover, if the spatial resolution of the SLS technology allows, the diffractive element could be built directly on the surface of the refractive one, ensuring corrections for some of the refractive optical element aberrations - high performance diffractively corrected refractive optics could emerge.

If polymers transparent to $\mathrm{THz}$ are made available for use with SLS, then one may envision other optical elements such as THz holograms - either surface or volume holograms - used for 3D patterning/structuring of the THz field for various applications. Moreover, multilayer interference filters may be built for the spectral analysis of the incident radiation. Such a filter and/or hologram can be built directly on the planar surface (base) of the lenses considered above, thus creating optical elements with a complex functionality - like in this particular example - spectral filtering and focusing. Optical elements for imparting an orbital momentum to the THz photon can also be made by SLS on such materials. Lenses of all kinds (spherical, aspherical, free form), dielectric reflectors of complex shapes and dielectric waveguides and antennas are other types of electromagnetic elements that can be considered for fabricating with SLS directly on THz transparent polymers and without the need of casting into mould.

Here, we would like to bring to the attention the thermoplastic TPX polymer. This material is transparent in a wide spectral range of the $\mathrm{THz}$ domain as well as in the visible part of the spectrum. It also absorbs at 10.6 microns, the wavelength of the $\mathrm{CO}_{2}$ laser, making it an interesting candidate for polymer SLS. In a more detailed approach, the $\mathrm{CO}_{2}$ laser is used to pump the molecular lasers emitting at wavelengths well above 20 microns. In this case, a TPX window is used as spectral separator, TPX being transparent to the lasing emission of the molecular laser, while avoiding outside propagation of the 10.6 micron pump beam. While its use to make visible optics by SLS is not appropriate because of both the poor resolution of SLS (compared to the wavelength of visible light of 0.5 microns) and of the microporous 
structure that will give rise to high optical scattering and foggy aspect in the visible spectrum, it will, however, represent a benefit when used in the THz region. As shown in this paper, such optical elements made by SLS behave very well in the $\mathrm{THz}$ region. The grain size will be determinant for the porosity of the selective laser sintered part and will ultimately dictate the upper THz frequency at which the material can work without excessive scattering. Moreover, TPX has a low refractive index of 1.46, making the optical element more tolerant to the effect of the staircase steps than polymers with higher index such as PA2200.

One of the main advantages of using SLS to make optical systems is the ability to make all the lenses and their support structures as one rugged object. This is especially useful when the respective optical system is subjected to strong mechanical stress and vibrations, one such example being encountered when launching an unmanned satellite to orbit. In the case of mechanical stress and vibrations, the optical system's support structure can be designed and optimized by using the finite element simulation methods for withstanding the stress without compromising the optical figure of merit of the THz optical system even in such adverse conditions.

In the following, we discuss other aspects related to the use of layer-by-layer additive manufacturing, particularly SLS, for making optical elements.

\section{a) Refractive or reflective optics?}

Until now, we have considered polymers that are used for building lenses and optical systems by selective laserr sintering. These are refractive optical elements. Another possibility is to use direct metal laser melting and create optics (mirrors) that is reflective for the $\mathrm{THz}$ range. This is even a better case because of two reasons:

- the reflective optics is intrinsically achromate and has the same optical transmission for all the wavelengths in the $\mathrm{THz}$ range, practically covering this entire part of the spectrum (up to tens of $\mathrm{THz}$ ).

- the direct metal laser melting technique works with layers of 20 microns thickness, which means that even shorter wavelengths can be accomodated withoit any distortion when compared to traditional mirrors. Considering the results above and the 20 microns step, the maximum working frequency - that is limited only by the step thickness and not by the optical properties - attains values of $10 \mathrm{THz}$.

Moreover, the as-made mirror or reflective, self-aligned THz optical system can be immersed in an electrolyte bath and the corners of the steps be smoothened by a slight electrochemical corrosion, thus improving the quality of the surface. This corner rounding can be followed by a finely tuned electrochemical polishing for creating a surface quality that further enhances the optical behaviour of the optics. A final step of metal electro- or vacuum deposition can be considered for passivating the surface against ambient conditions and for ensuring better reflectivity. While many metals can be used for this purpose that have similar reflectivities in the THz range (e.g., $\mathrm{Au}, \mathrm{Ag}, \mathrm{Al}$ ), $\mathrm{Gold}$ is the best one when considering also surface passivation needs. For space applications, such passivation is not needed.

\section{b) Roughness and porosity effect}

Except of the stepwsie structure, another characteristic feature of additive manufacturing achieved by selective laser sintering is surface roughness of the as-made parts. This peculiarity may have different effects on the optical behaviour of the lens, depending on the concrete roughness value (process dependent) and on the incident radiation wavelength. A typical value range for roughness in the case of polymer based SLS is of few tens of microns [38]. For having an influence on the incident $\mathrm{THz}$ radiation, the roughness values must be greater than a tenth of the wavelength value. Thus, for a roughness of 22 microns, the minimum radiation wavelength at which the roughness has no effect is of 220 microns (in vacuum), corresponding to a frequency of $1.36 \mathrm{THz}$. We thus have several frequency intervals in which $\mathrm{THz}$ radiation behaves differently at the air-lens interface.

In the lower $\mathrm{THz}$ frequency range, where wavelengths are of the order of millimetre, the roughness plays practically no role and the air-lens interface can be considered smooth. In this case, the lens behaviour does not get affected. Because of the sharp interface, a certain THz radiation reflection will occur, contributing to signal loss.

Going to higher frequencies, there is an intermediate frequency range at which the interface behaves as a metamaterial having an effective refractive index given by the polymer electric permittivity and the polymer volume filling ratio (at the interface). Obviously, this refractive index is lower at the air interface and increases towards the bulk. This happens at frequencies between $1.36 \mathrm{THz}$ and $3 \mathrm{THz}$ and, in this case, the roughness layer plays the role of an antireflection coating. Thus, we expect that the reflection coefficient (and associated losses) be lower in this frequency range. As a cautionary note, the frequencies indicated in this paragraph are not of strict values, they are mostly for orientation purposes, the passage from one regime to the other taking place gradually.

At even higher frequencies (shorter wavelengths) the roughness cannot be considered as the only aspect hindering the radiation-lens interaction at the interface with air. The granular structure of the surface begins to play a major role and 
the averaged, metamaterial-like behaviour ceases to exist; local structure starts to influence radiation interaction and scattering appears.

A similar consideration occurs for material porosity. In this case, the material behaves as a metamaterial for frequencies up to a few THz. Above that frequency, the granular structure becomes 'visible' to the radiation and scattering and radiation localization / trapping take place as well. During radiation trapping in some of the material's granules, localized thermally-based nonlinear optical effects can take place since radiation trapping will give rise to a local intensity increase up to the point when material might soften or even change its optical properties in a thermally-dependent way.

It is thus obvious that additive manufacturing of optical components cannot be used in the higher frequency part of the $\mathrm{THz}$ spectrum even if they are transparent just because of surface roughness and volume porosity.

However, things can be improved in several ways. The first one is to use a higher temperature in the work chamber of the SLS system, for example $170^{\circ} \mathrm{C}$ in the case of PA2200. Reducing laser scan speed could also be a choice, alone or together with the temperature in the work chamber. In this case it must be considered the fact that, being a bulk element, the lens will contract significantly during the post-process cooling period and also have some warping. Geometrical accuracy might be compromised if the CAD model is not carefully designed to consider these processes. The second way of dealing with porosity is to use the polymer as a mould and then, by double replication (double-inversion), to create the non-porous $\mathrm{THz}$ lens by casting. For this case, the initially additively manufactured lens is used as a mould for a temperature withstanding material (micronized gypsum, for example) and a first replica of it is made. Then, the gypsum replica is used as a mould for the same or other THz transparent polymer that is melt and then poured into it. A third option is to use Direct Metal Laser Sintering or Selective Laser Melting for making the mold, a technique similar to SLS but using metal instead of polymer. In this case, the layer thickness (surface step height) may attain values as low as 20 microns, which clearly gives better surface finish for the final polymer $\mathrm{THz}$ lens. The molten polymer is poured into this metal mold and let to solidify.

\section{c) Influence of polymer thermal expansion}

The thermal expansion plays a role in several stages of the fabrication: first, during the post-SLS cooling and then during the exploitation of the lens. For the post-SLS stage, careful CAD design should be performed, preferably by using calibrated data. Calibrated data can be obtained by manufacturing several versions of the lens and then determining the variation from the desired geometry. Based on these data that consider all these effects, a better version of the CAD model can thus be built.

The thermal expansion may play a role during exploitation only if the lens is used in an environment in which temperature varies significantly. While the refractive index changes with temperature, the most important variation, especially in the case of (semi)crystalline polymers, comes from their thermal expansion. For example, PA2200 expands ten times more than most of the metals for the same temperature difference. Here additive manufacturing may play a very important role not only on single optical elements, but also on full optical systems. To control thermal expansion, thermal stabilization channels can be fabricated in the support structure of the optical system / lens. Fluids may flow through these channels ensuring the thermal stabilization of the entire system. Such channels can be a mix of solutions: one set of channels can be made for liquids that thermally stabilize the support structure while a separate set of channels is used for blowing air (or other gas) onto the lens surface for controlling and stabilizing its temperature. A more efficient air blowing system may use the Coandă effect that will ensure the full exposure of the lens to the gas jet provided that the gas flow is laminar. The laminar flow is obtained by carefully designed gas outlets that can be easily fabricated by additive manufacturing. In this way additive manufacturing brings one more advantage to the fabrication of $\mathrm{THz}$ optical systems by ensuring an efficient thermal management for special applications and for the cases when the $\mathrm{THz}$ optical system operates in a harsh environment.

\section{d) Only passive THz optical elements?}

Up to now we have discussed only passive optical elements and systems, that is systems whose properties / behaviour cannot be changed in an active, controlled manner. Passive systems use polymers that, at least to the best of our knowledge, do not have nonlinear optical properties that could be controlled either electrically or magnetically, at least not in their bulk form. However, there are two ways of achieving e certain tunability for a $\mathrm{THz}$ optical system made by additive manufacturing. The first way is to use temperature as a control parameter for hanging the optical behaviour of the system. While being feasible, especially if we take into account what we have discussed above at point b), it is a slow process with a limited range of tunability. The other way is to use some fluid channels for injecting or extracting various fluids with well-defined $\mathrm{THz}$ properties. The fluids can be inserted either in hollow optical elements changing their refractivity or in the space between such elements, thus replacing the air in between. This process is faster and it allows a 
wide range of tunability. As mentioned above, additive manufacturing offers the unique capability to easily integrate mechanical, optical and fluid transport devices in one stand-alone system.

It is worth mentioning another possible solution. This consists in replacing the plain polymer with a polymer composite that has the filling made of materials that either change their properties in response to electric/magnetic fields or allow an electromagnetic actuation of the optical element (mechano-optical tuning). While this is a possible approach, such composites should be first characterized with respect to their behaviour to $\mathrm{THz}$ radiation as well as their suitability to be processed by SLS and only after that make any further assertions.

Two important applications of this work should be mentioned here. These applications are empowered by better, high quality imaging possibilities obtained at affordable costs (no tedious alignment of optical elements is necessary) and also by the possibility to make some of the preliminary image processing in an all-optical manner. One example of the latter is the all-optical Fourier transform made to the incident image or incident time-varying signal (see [35-37]). Additive manufacturing offers the possibility to make such complex, data processing THz optical systems.

The first SLS application is in the field of ultrafast communications. As it is known, higher data rates and higher number of simultaneous communications channels require a higher frequency of the carrier electromagnetic wave. Such an additively manufactured THz optical system may be placed aboard a satellite that is able to handle frequencies in that range and ensure a proper distribution of the communication channels both for gathering the incoming signals and for their further sending to destinations while improving their quality / intensity distribution in an on-flight, real-time manner. A rugged, small footprint THz optical system is essential for such applications. It is of great importance to make the optical system in a single manufacturing process without any subsequent optical alignment and mounting.

The second application is in the medical imaging domain where a high-performance $\mathrm{THz}$ imaging system based on optical elements with complex geometries can be made only by SLS. Optical systems with complex geometries may bring improved diagnosis capabilities for a wide range of health problematics, starting with the diagnosing of skin tissue and going to remote, non-contact sweat and exhalation analysis (spectroscopy). Also in this case, all-optical processing of the incoming THz signal / image (e.g., optical Fourier transform of the image) empowers the use of THz radiation for this application.

Another, special application is in space exploration, more precisely in astrobiology. Having a rugged, small footprint THz optical system may help in-situ study of complex molecules by THz spectroscopy and imaging. Such an exploration robot could use this capability for searching for signs of life on other celestial bodies. As it is known, large complex molecules, some of them associated with life, have a rich spectrum in the THz spectral domain and thus this particular range of electromagnetic spectrum can be very useful for their detection. THz spectroscopy is also sensitive enough to the isotopic composition of the molecules (at least for hydrogen isotopes). This is important since the isotopic mass variation changes the eigenfrequencies of the molecule's vibration modes. An unusual isotopic ratio could eventually signal a possible form of life or a rare non-biotic process. All the above entitles us to state that additive manufacturing could play an important role in astrobiology studies. Needless to say is the fact that this comes together with the ability of (microscale) additive manufacturing to fabricate complex fluidic microlabs and lab-on-chip for such in-situ exploration missions.

Finally, we mention that our results and considerations confirm earlier findings, see for example [28], [39].

\section{Conclusion}

In this paper we have shown that the stair-like structure specific to SLS process is not critical when considering the additive fabrication of the $\mathrm{THz}$ optical elements. It becomes challenging when the frequencies are above $1.5 \mathrm{THz}$, when shorter wavelengths "sense" more acutely the effect of steps and of material porosity. The use of polymers that are transparent in this spectral range allows the creation of very complex optical elements that enable a finer manipulation of $\mathrm{THz}$ radiation and extend the area of applications of this part of the electromagnetic spectrum. Also important, the use of $\mathrm{THz}$ transparent polymers in combination with SLS allows to additively manufacture a whole rugged, self-aligned optical system. A clever design of the optical system with a small footprint and good mechanical strength could offer the possibility to use it in space-borne ships for applications such as ultrafast communications, $\mathrm{THz}$ astronomy and astrobiology, among others.

\section{Acknowledgements}

The work was performed under the framework of the Structural Funds contract No. 77/2016 "Parteneriat în exploatarea Tehnologiilor Generice Esenţiale (TGE), utilizând o PLATformă de interacţiune cu întreprinderile competitive (TGE-PLAT)", Cod MySMIS 105623, sub-contract 77.5D - Sistem optic formator de imagine folosind componente "freeform" $(F F)$ şi tehnologie de realizare a acestora, POC-A1-A1.2.3-G-2-15 1.2.3. Parteneriat pentru transfer de 
cunoştinţe. The authors gratefully thank dr. Titus Sandu from IMT-Bucharest for carefully reading and commenting on the manuscript.

\section{Authors' contribution}

G.M.P. devised the work, coordinated it, devised the concept for as-made self-aligned, channel-integrated THz optical systems and prepared the manuscript. C.T. performed the EM simulations and verified the manuscript. All the authors agreed to the manuscript.

\section{References}

[1] K. I. Zaytsev, I. N. Dolganova, N. V. Chernomyrdin, G. M. Katyba, A. A. Gavdush, O. P. Cherkasova, G. A. Komandin, M. A. Shchedrina, A. N. Khodan, D. S. Ponomarev, I. V. Reshetov, V. E. Karasik, M. Skorobogatiy, V. N. Kurlov, V. V. Tuchin - "The progress and perspectives of terahertz technology for diagnosis of neoplasms: a review", J. Opt. vol. 22, p. 013001, (2020)

[2] P. Weightman - "Prospects for the study of biological systems with high power sources of terahertz radiation", Phys. Biol. vol. 9, p. 053001, (2012)

[3] J. A. Zeitler, L. F. Gladden - "In-vitro tomography and non-destructive imaging at depth of pharmaceutical solid dosage forms", Eur. J. Pharm. Biopharm. vol. 71, p. 2-22, (2009)

[4] B. B. Hu, M. C. Nuss - "Imaging with terahertz waves", Optics Letters vol. 20, no. 16, p. 1716-1718, (1995)

[5] D.M. Mittleman, M. Gupta, R. Neelamani, R.G. Baraniuk, J.V. Rudd, M. Koch - "Recent advances in terahertz imaging", Applied Physics B, vol. 68, p.1085-1094, (1999)

[6] A. Vella, J. Houard, L. Arnoldi, M. Tang, M. Boudant, A. Ayoub, A. Normand, G. Da Costa, A. Hideur - "Highresolution terahertz-driven atom probe tomography", Sci. Adv. 7 : eabd7259, (2021)

[7] P. U. Jepsen, B. M. Fischer - "Dynamic range in terahertz time-domain transmission and reflection spectroscopy", Opt. Lett. vol. 30, p. 29-31, (2005)

[8] M. Tonouchi - "Cutting-edge terahertz technology", Nat. Photon. vol. 1, p. 97-105, (2007)

[9] P. U. Jepsen, D. G. Cooke, M. Koch - "Terahertz spectroscopy and imaging-modern techniques and applications", Laser Photon. Rev. vol. 5, p. 124-166, (2011)

[10] Christopher K. Walker - Terahertz Astronomy, CRC Press, ISBN: 9781466570436, (2016)

[11] V. Minier, G. Durand, P.-O. Lagage, M. Talvard - "CAMISTIC: THz/submm astronomy at Do me C in Antarctica", Highlights of Astronomy, volume 14IAU XXVI General Assembly, Karel A. van der Hucht, ed., p. 709-710, (2006)

[12] Th. de Graauw - "THz Astronomy from Space", In: Chamberlain J.M., Miles R.E. (eds) New Directions in Terahertz Technology. NATO ASI Series (Series E: Applied Sciences), vol 334. Springer, Dordrecht. https://doi.org/10.1007/978-94-011-5760-5_19, (1997)

[13] C. Corsi, F. Sizov (eds.) - "THz and Security Applications - Detectors, Sources and Associated Electronics for THz Applications", Springer Science+Business Media Dordrecht, ISBN 978-94-017-8827-4, (2014)

[14] X. C. Zhang, J. Xu - "THz Technology in Security Checks", In: Introduction to THz Wave Photonics. Springer, Boston, MA. https://doi.org/10.1007/978-1-4419-0978-7_9, (2010)

[15] I. V. Minin, O. V. Minin - "THz quasioptics applications in security", Proc. of SPIE Vol. 6212 p. 621210 (12 pages), (2006)

[16] V. L. Vaks, V. A. Anfertev, V. Y. Balakirev, S. A. Basov, E. G. Domracheva, A. V. Illyuk, P. V. Kupriyanov, S. I. Pripolzin, M. B. Chernyaeva- "High resolution terahertz spectroscopy for analytical applications", Physics Uspekhi vol. 63, no. 7, p. $708-720,(2020)$

[17] F. Rutz, M. Koch, S. Khare, M. Moneke, H. Richter, U. Ewert - "Terahertz quality control of polymeric products", International Journal of Infrared and Millimeter Waves, volume 27, p. 547-556, (2006)

[18] K. Ahi, S. Shahbazmohamadi, N. Asadizanjani - "Quality control and authentication of packaged integrated circuits using enhanced-spatial-resolution terahertz time-domain spectroscopy and imaging", Optics and Lasers in Engineering, vol. 104, p. 274-284, (2018)

[19] S. S. Dhillon et al - "The 2017 Terahertz science and technology roadmap", J. Phys. D: Appl. Phys., vol. 50, p. 043001 (49pp), (2017)

[20] A. Dobroiu, C. Otani, K. Kawase - "Terahertz-wave sources and imaging applications", Meas. Sci. Technol., vol. 17, p. R161-R174, (2006)

[21] C. Jansen, S. Wietzke, O. Peters, M. Scheller, N. Vieweg, M. Salhi, N. Krumbholz, C. Jördens, T. Hochrein, M. Koch

- "Terahertz imaging: applications and perspectives", Applied Optics, vol. 49, no. 19, p. E48-E57, (2010)

[22] H. A. Hafez, X. Chai, A. Ibrahim, S. Mondal, D. Férachou, X. Ropagnol, T. Ozaki - "Intense terahertz radiation and their applications", J. Opt., vol. 18, p. 093004 (48pp), (2016)

[23] W. L. Chan, J. Deibel, D. M. Mittleman - "Imaging with terahertz radiation", Rep. Prog. Phys., vol. 70, p. 13251379, (2007) 
[24] B. Ung, A. Mazhorova, A. Dupuis, M. Rozé, M. Skorobogatiy - "Polymer microstructured optical fibers for terahertz wave guiding", Optics Express, vol. 19, no. 26, B848, (2011)

[25] T. Kaji, Y. Tominari, T. Yamada, S. Saito, I. Morohashi, A. Otomo - "Terahertz-wave generation devices using electro-optic polymer slab waveguides and cyclo-olefin polymer clads", Optics Express, vol. 26, no. 23, p. 30466-30475, (2018)

[26] D. Chen, H. Chen - "A novel low-loss Terahertz waveguide: Polymer tube”, Optics Express, vol. 18, Issue 4, p. 3762$3767,(2010)$

[27] J. Yuan, T. Ning, H. Li, L. Pei, J. Li, J. Zheng, L. Wan - "Terahertz filters based on subwavelength polymer waveguide", Results in Physics, volume 13, p. 102198, (2019)

[28] S. F. Busch, M. Weidenbach, J. C. Balzer, M. Koch - "THz Optics 3D Printed with TOPAS", J. Infrared Milli. Terahz. Waves, (2015) DOI 10.1007/s10762-015-0236-7

[29] K. Szkudlarek et al - "Terahertz 3D printed diffractive lens matrices for field-effect transistor detector focal plane arrays", Optics Express, vol. 24, Issue 18, p. 20119-20131, (2016)

[30] T. Yu, X. Zuo, W. Liu, C. Gong - "0.1THz super-resolution imaging based on 3D printed confocal waveguides", Optics Communications, vol. 459, p. 124896, (2020)

[31] S. F. Busch, M. Weidenbach, M. Fey, F. Schäfer, T. Probst, M. Koch - "Optical Properties of 3D Printable Plastics in the $\mathrm{THz}$ Regime and their Application for 3D Printed THz Optics", J. Infrared Milli. Terahz. Waves, vol. 35, p. 993-997, (2014)

[32] K. Szkudlarek, M. Sypek, G. Cywiński, J. Suszek, P. Zagrajek, A. Feduniewicz-Żmuda, I. Yahniuk, S. Yatsunenko, A. Nowakowska-Siwińska, D. Coquillat, D. B. But, M. Rachoń, K. Węgrzyńska, C. Skierbiszewski, W. Knap - "Terahertz 3 D printed diffractive lens matrices for field-effect transistor detector focal plane arrays", Optics Express vol. 24, no. 18, p. $20119-21131,(2016)$

[33] B. Ung, J. Balakrishnan, B. Fischer, B. W.-H. Ng, D. Abbott - "Terahertz detection of substances for security related purposes", Proc. of SPIE vol. 6414, p. 64141U1 - 64141U6, (2007)

[34] V. E. Rogalin, I. A. Kaplunov, G. I. Kropotov - "Optical Materials for the THz Range", Optics and Spectroscopy, vol. 125, no. 6, p. 1053-1064, (2018); original Russian version published in Optika i Spektroskopiya, vol. 125, no. 6, p. $851-863,(2018)$.

[35] J. Goodman - Introduction to Fourier Optics (3rd ed.), Roberts \& Company Publishers, (2005). ISBN 0-9747077-24

[36] K. Lee, C. T.D. Thai, J.-K. Kevin Rhee - "All optical discrete Fourier transform processor for 100 Gbps OFDM transmission", Optics Express, vol. 16, issue 6, p. 4023-4028, (2008)

[37] D. Hillerkuss, M. Winter, M. Teschke, A. Mărculescu, J. Li , G. Sigurdsson, K. Worms, S. Ben Ezra, N. Narkiss, W. Freude, J. Leuthold - "Simple all-optical FFT scheme enabling Tbit/s real-time signal processing", Optics Express vol. 18, no. 9 , p. $9324-9340,(2010)$

[38] S. Petzold, J. Klett, A. Schauer, T. A. Osswald - "Surface roughness of polyamide 12 parts manufactured using selective laser sintering", Polymer Testing, v, 80, p. 106094, (2019)

[39] B. Zhang, W. Chen, Y. Wu, K. Ding, R. Li - "Review of 3D Printed Millimeter-Wave and Terahertz Passive Devices", International Journal of Antennas and Propagation vol. 2017, Article ID 1297931, 10 pages, (2017) https://doi.org/10.1155/2017/1297931 


\section{Supplementary Files}

This is a list of supplementary files associated with this preprint. Click to download.

- SLSTHzOpticalSystemsSUPPLEMENTALMATERIAL.pdf 